\title{
A Sequential Selection Game with Vetoes CDAM-2008-19
}

\author{
Steve Alpern, Shmuel Gal, Eilon Solan
}

July 10, 2008

\begin{abstract}
We study an on-line selection game between two committee members (the players). The committee interviews candidates sequentially and has to decide, after each interview, whether to hire or to interview the next candidate.. Each player can either accept or reject the candidate, and if he rejects the candidate while the other accepts her, he can cast a veto. The candidate is hired if accepted by at least one player and not vetoed. The total number of vetoes available for each player is fixed in advance.

We prove the existence of a subgame perfect equilibrium for this game if there are a finite number of candidates types. For a general candidate distribution we prove the existence of an $\varepsilon-$ subgame perfect equilibrium. We also demonstrate several unusual phenomena, including situations in which a player prefers the other player would have an extra veto, and even prefers to give his own veto right to the other player.
\end{abstract}

\section{Introduction}

Candidates for various positions, both in the private and public sectors, are often chosen by committees. When the decision regarding which candidate to accept is made after all the candidates have been interviewed, an issue regarding the design of the selection rule arises: the impossibility theorem of Gibbard (1973) and Satherwaite (1975) states that there is no rule that the committee can use to select the best candidate and that satisfies several desirable properties. 
Sometimes the committee interviews the candidates sequentially ('online') and has to decide, after each interview, whether the current candidate is acceptable or not; in the latter case, the candidate "disappears", and cannot be selected in the future if it turns out that she has been the best candidate. Such a case occurs, e.g., in the selection of juries in the American legal system, in which both sides have the right to veto candidates, or when a couple looks for an apartment to rent when demand far exceeds supply, and apartments are rented within hours.

In the present paper we study an on-line selection game between two committee members, who interview candidates sequentially and have to decide, after each interview, whether to accept the current candidate or to reject her. We model the process by which the committee decides on each candidate as follows. First one of the committee members, the leader, decides whether to accept or reject the candidate, and then the second member, the follower, observing the decision of the leader, decides whether to accept or reject the candidate. If both members rejected the candidate then she is rejected; if both members accepted the candidate then she is accepted; if one of the candidates accepted the candidate while the other rejected her, the member who rejected the candidate has the right to cast a veto, in which case the candidate is rejected. If no veto was cast, the candidate is hired. We assume that the number of vetoes of each member is given in advance, as occurs, e.g., in jury selection (see Brams and Davis (1976), (1978) and DeGroot and Kadane (1980)). In many cases the number of vetoes that a member has is not fixed explicitly, yet, since successive vetoes often raise pressure from other committee members against more vetoes. Thus, the number of vetoes of a member measures his relative strength, both his mental strength and his position within the organization.

Each candidate is characterized by two numbers, her utility to each committee member, which is termed the type of the candidate. The full type is known to both players. The goal of each player is to maximize his own expected utility from the selected candidate.

Our model is a game theoretic extension of the well known "secretary problem" (see, e.g., Ferguson (1989, 2001), Eriksson et al (2007)), in which quality, rather than rank of quality, is optimized, and each player's utility is his evaluation of the hired candidate. A different game variant of the "secretary problem", in which various employers compete on employees who are observed sequentially, was studied by, e.g., Enns and Ferenstein (1987), Ramsey and Szajowski (2005) and Sakaguchi (2005). 
Our model is also related to the selection problem studied in DeGroot and Kadane (1980). The main difference is that whereas they study jury selection problems, and therefore allow only two actions (veto or no veto), we study an employee selection problem, and therefore allow three actions (accept, reject with veto, and reject without veto). As we show below, the two models exhibit quite a different behavior.

Our model was introduced in Alpern and Gal (2008), who proved that if a player's veto rights increase (while the other's remains fixed), then his equilibrium payoff cannot decrease. Thus, the number of vetoes each player has at the beginning of the game measures, in a sense, his relative strength. Alpern and Gal (2008) also presented a detailed analysis of this game for uniformly distributed candidate types, and established the existence of equilibria in threshold strategies for the game with no vetoes. Additionally, they study a discounted version of the game, and in this case they determined the optimal number of vetoes that a social planner should give the players, if the social planner's goal is to maximize the expected sum of utilities of both players.

In the present paper we show that when there are finitely many types of candidates an equilibrium in Markovian strategies always exists, while when there are infinitely many types of candidates, an equilibrium may fail to exist, yet an $\varepsilon$-equilibrium in Markovian strategies exists for every $\varepsilon>0$. We then study the structure of Markovian equilibria, distinguishing between two types of equilibria: progressive equilibria and helpful equilibria. In a progressive equilibrium each player prefers that the other player would have fewer vetos, and even accepts bad (to him) candidates in order to force the other player to "waste" a veto. In a helpful equilibrium players prefer the other to have more vetos and hence reject candidates that the other player would otherwise have to veto.

We also present surprising phenomena that may occur in our games. DeGroot and Kadane (1980) present an example of selecting a jury of two participants, in which one of the committee members prefers to give his veto right to the other member. This phenomenon is called the "paradox of redistribution" by Fischer and Schotter (1978) or the "donation paradox" (see Felsenthal and Machover (1995), Kadane et al. (1999), and Holler and Napel (2004)). DeGroot and Kadane (1980, Theorem 3) prove that if the committee has to choose a single candidate, then the donation paradox cannot occur. Surprisingly, as we show by an example, a donation paradox may occur in our game. We also provide an example that shows that a worse population 
(good candidates are rarer) may yield a better outcome.

The paper is organized as follows. Section 2 describes the model. Section 3 proves the existence of a subgame perfect equilibrium $(S P E)$, in mixed Markovian strategies. Section 4 describes some counter-intuitive behavior of the game with no vetoes, including the donation paradox. Section 5 describes the structure of the equilibria in the game. We use a simple three atom distribution to demonstrate the possible types of equilibria, and to show that a player may prefer that the other player is given an extra veto, even in the worth of a candidate to one player is independent of her worth to the other player.

\section{The Model}

We consider a sequential two-player selection game $G(i, j)$, that is given by two non-negative integers $i$ and $j$ (the number of vetoes of the two players) and a distribution $F_{2}$ on the unit square (the distribution of candidate). At each stage $t$, if the game has not terminated before, the players play the extensive form game that appears in Figure 1: first Nature chooses a candidate $(x, y) \in[0,1]^{2}$ according to the distribution $F_{2}$, and then player I decides whether to Accept or to Reject the candidate. If player I decides to accept the candidate, and if $j>0$, player II decides whether to Veto the candidate, and then from the next stage on the game $G(i, j-1)$ is played, or whether Not to Veto the candidate, in which case the game terminates with a terminal payoff $x$ to player I and $y$ to player II. If player I decides to Reject the candidate, player II decides whether to Accept or to Reject the candidate. If player II decides to reject the candidate, the stage ends, and the game continues to the next stage. If player II decides to accept the candidate, player I decides whether to Veto the candidate, and then from the next stage on the game $G(i-1, j)$ is played, or whether Not to Veto the candidate, in which case the game terminates with a terminal payoff $(x, y)$. If no candidate is ever accepted the payoff to both players is 0. In Figure 1, the actions Accept, Reject, Veto and No Veto are abbreviated to a, r, v and $n$ respectively. 


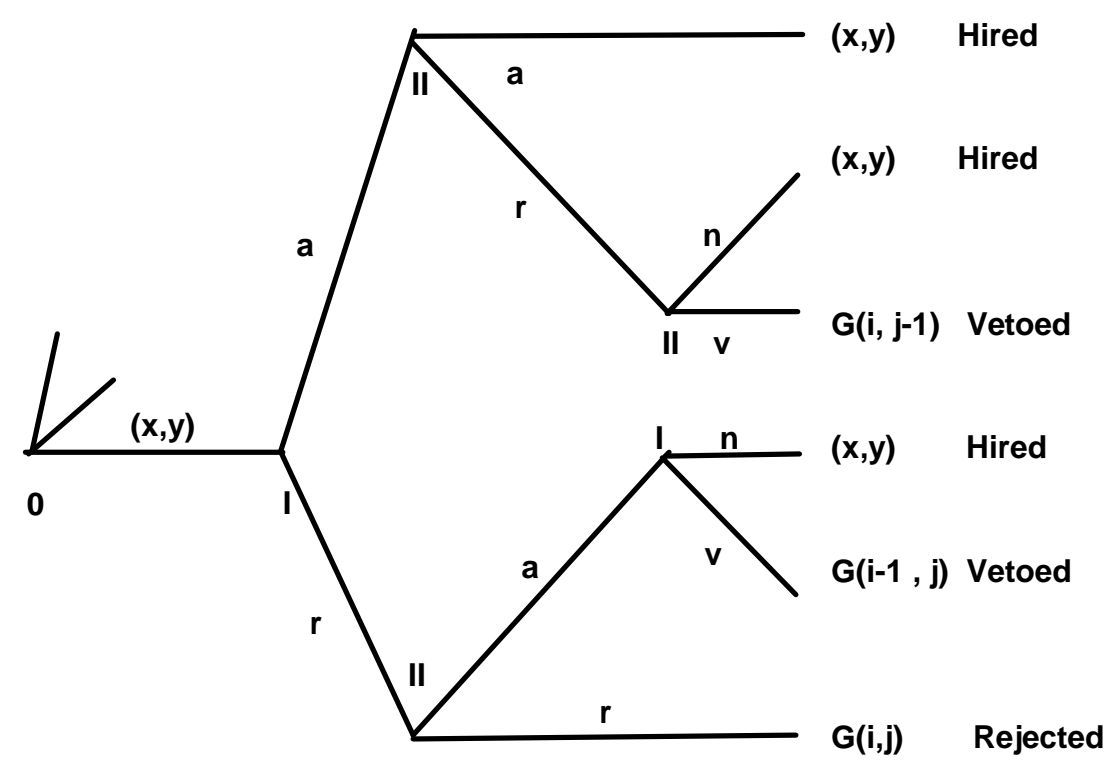

Figure 1. Reduced tree for $G(i, j)$

A strategy for a player is a rule that indicates how to play at each stage given any past history. A strategy is Markovian if the mixed action to be played after every given history depends only on the type of the current candidate and on the number of vetoes left for both players.

Since we study Markovian strategies, the continuation payoffs in $G(i, j-1)$ and $G(i-1, j)$ are independent of past play. We denote the continuation payoff in $G(i, j-1)$ by $(u+, v-)$ and the continuation payoff in $G(i-1, j)$ by $(u-, v+)$.

Every pair of strategies $(\sigma, \tau)$ induces an expected payoff for each player $k \in\{I, I I\}$, which we denote by $\gamma^{k}(\sigma, \tau)$. For every $\varepsilon \geq 0$, an $\varepsilon$-equilibrium $(\sigma, \tau)$ is a pair of strategies such that no player can profit more than $\varepsilon$ by deviating. A pair of strategies is a subgame-perfect $\varepsilon$-equilibrium if for every finite history, the pair of strategies, restricted to the continuation game after this history occurs, is an $\varepsilon$-equilibrium.

Since the game is a game of perfect information (that is, there are no simultaneous moves, and all past moves are observed), it follows from Mertens (1987) that for every $\varepsilon>0$ an $\varepsilon$-equilibrium exists. The construction of 
Mertens (1987) uses threats of punishment, and therefore it does not yield a subgame-perfect $\varepsilon$-equilibrium.

\section{Existence of a Subgame Perfect Equilib- rium}

Let $W \subset[0,1]^{2}$ be the support of $F_{2}$, that is, the set of all possible types $(x, y)$ of the candidates. When $(x, y)$ is the type of a candidate, $x$ (resp. $y$ ) is the worth of the candidate for player I (resp. II). We make the following assumption throughout.

A The types of the candidates in different stages are i.i.d.

The following example shows that in general a subgame perfect 0 -equilibrium may not exist.

Example 1 Suppose $F_{2}$ is the uniform distribution in the polytope whose extreme points are $(0,0),(0, c),(c, 0),(1,1)$, where $0<c<\frac{1}{2}$. In $G(0,0)$, the strategy pair in which each player accepts a candidate once her worth for him is at least $1-\varepsilon$ is a subgame perfect $\varepsilon$-equilibrium. We claim that the game $G(0,0)$ has no 0-equilibrium. Indeed, a 0-equilibrium must be in threshold strategies: accept a candidate if her worth for you is above a given threshold $\alpha$, reject it if her worth for you is below the threshold $\alpha$, and possibly mix if her worth for you is equal to the threshold $\alpha$. However, if one player uses such a threshold strategy with threshold $\alpha$ (and $\alpha<1$ ), then a simple calculation shows that the other player's best reply is a threshold strategy with threshold strictly larger than $\alpha$. This implies that the only candidate for a 0-equilibrium is when the threshold is 1, but then with probability 1 no player ever accepts a candidate (and the payoff is 0 to both players).

Our main result in this section is the following.

Theorem 2 The game $G(i, j)$ admits a subgame-perfect $\varepsilon$-equilibrium in Markovian strategies for every $\varepsilon>0$. If $F_{2}$ has finite support, there is a subgame-perfect 0-equilibrium in Markovian strategies.

The proof of this theorem is done in several lemmas. We first prove that to prove the first claim of Theorem 2 it is sufficient to prove its second part. 
Lemma 3 Suppose that whenever $F_{2}$ has finite support the game $G(i, j)$ admits a subgame perfect 0-equilibrium in Markovian strategies. Then for every $F_{2}$ (with general support) the game admits a subgame perfect $\varepsilon$-equilibrium in Markovian strategies, for every $\varepsilon>0$.

Proof. We prove the Lemma by induction on $i+j$. We assume that $G(i-1, j)$ and $G(i, j-1)$ admit a subgame perfect $\frac{\varepsilon}{4}$-equilibrium in Markovian strategies for every $\varepsilon>0$, and we denote by $(u-, v+)$ and $(u+, v-)$ corresponding $\frac{\varepsilon}{4}-$ equilibrium payoffs in Markovian strategies in these games. To accommodate the case in which a player has no vetoes, if $i=0$ we set $u-=-1$, and if $j=0$ we set $v-=-1$.

Consider now the game $G(i, j)$. Let $a(x, y)$ be the absorbing payoff if player I accepts $(x, y)$; it is either $(x, y)$ or $(u+, v-)$, depending on which one player II prefers

$$
a(x, y)=\left\{\begin{array}{cc}
(x, y) & y \geq v- \\
(u+, v-) & y<v-
\end{array} .\right.
$$

Let $b(x, y)$ be the absorbing payoff if player I rejects $(x, y)$ and player II accepts it; it is either $(x, y)$ or $(u-, v+)$, depending on which one player I prefers:

$$
b(x, y)=\left\{\begin{array}{cc}
(x, y) & x \geq u- \\
(u-, v+) & x<u-
\end{array} .\right.
$$

Note that the only discontinuities of $a(x, y)$ and $b(x, y)$ are near the horizontal intervals $I_{x}=\{(x, v-), 0 \leq x \leq 1\}$ and $I_{y}=\{(u-, y), 0 \leq y \leq 1\}$

Suppose $F_{2}$ has a general support (not necessarily finite). Let $\widehat{F}_{2}$ be an $\frac{\varepsilon}{4}$-discretization of $F_{2}$. We wish to maintain continuity within each of our 'elements', so we first split $[0,1]^{2}$ into the following nine parts. $\{(x, y), u-<x \leq 1, v-<y \leq 1\},\{(x, y), u-<x \leq 1,0 \leq y<v-\}$, $\{(x, y), 0 \leq x<u-, v-<y \leq 1\},\{(x, y), 0 \leq x<u-, 0 \leq y<v-\}$ $\{(x, v-), 0 \leq x<u-\},\{(x, v-), u-<x \leq 1\},\{(u-, y), 0 \leq y<v-\}$, $\{(u-, y), v-<y \leq 1\}$ and $(u-, v-)$. Then, we partition each part (except the last part which is a single point) into finitely many sets such that each of the overall $L$ resulting sets $A_{1}, A_{2}, \ldots, A_{L}$, has diameter at most $\frac{\varepsilon}{4}$ :

$$
\sup _{(x, y),\left(x^{\prime}, y^{\prime}\right) \in A_{l}} d\left((x, y),\left(x^{\prime}, y^{\prime}\right)\right) \leq \frac{\varepsilon}{4}, \quad \forall l,
$$


and for each $l, 1 \leq l \leq L$, there is $\left(x_{l}, y_{l}\right) \in A_{l}$ such that $\widehat{F}_{2}\left(x_{l}, y_{l}\right)=F_{2}\left(A_{l}\right)$.

We are going to consider three games, $G_{1}, G_{2}$, and $G_{3}$. These are recursive absorbing games, in which at every stage a candidate is chosen according to some distribution, then player I, upon observing the candidate's type, decides whether to accept the candidate or reject it, and then player II, after observing the candidate's type and player I's decision, decides whether to accept it or reject it. The game terminates once at least one player accepts a candidate (there is no opportunity to cast vetoes), and the games only differ in the underlying distribution according to which candidates are chosen and by their absorbing payoffs.

- In the game $G_{1}$ the candidates' distribution is $F_{2}$ and the absorbing payoffs $a(x, y)$ (resp. $b(x, y))$ if $(x, y)$ is accepted by player I (resp. player II).

- In the game $G_{2}$ the candidates' distribution is $F_{2}$. If $(x, y) \in A_{l}$ is accepted by player I (resp. player II), the absorbing payoffs is $a\left(x_{l}, y_{l}\right)$ $\left(\right.$ resp. $\left.b\left(x_{l}, y_{l}\right)\right)$.

- In the game $G_{3}$ the candidates' distribution is $\widehat{F}_{2}$ and the absorbing payoffs is $a\left(x_{l}, y_{l}\right)$ (resp. $\left.b\left(x_{l}, y_{l}\right)\right)$ if $(x, y) \in A_{l}$ is accepted by player I (resp. player II).

Note that a $\frac{3 \varepsilon}{4}$-equilibrium in the game $G_{1}$ can be turned into an $\varepsilon$ equilibrium in $G(i, j)$, by supporting it with $\frac{\varepsilon}{4}$-equilibria in $G(i-1, j)$ and $G(i, j-1)$ that yield payoff $(u-, v+)$ and $(u+, v-)$ respectively if one of the players casts a veto. Denote by $\gamma_{1}(\sigma, \tau), \gamma_{2}(\sigma, \tau)$ and $\gamma_{3}(\sigma, \tau)$ the payoff functions in these three games. Since the absorbing payoffs in $G_{1}(i, j)$ and in $G_{2}(i, j)$ differ by at most $\frac{\varepsilon}{4}$, we have

$$
\left\|\gamma_{1}(\sigma, \tau)-\gamma_{2}(\sigma, \tau)\right\| \leq \frac{\varepsilon}{4}, \quad \forall \sigma, \forall \tau
$$

Fix a strategy pair $(\sigma, \tau)$. Let $\sigma_{3}$ by the strategy that is defined as follows:

$$
\sigma_{3}(x, y)=\frac{\int_{A_{l}} \sigma\left(x^{\prime}, y^{\prime}\right) d F_{2}\left(x^{\prime}, y^{\prime}\right)}{F_{2}\left(A_{l}\right)} \quad \text { if }(x, y) \in A_{l} .
$$

This is the average probability to accept $(x, y)$ under $\sigma$, provided it is in $A_{l}$. We define this strategy only for $(x, y) \in A_{l}$ such that $F_{2}\left(A_{l}\right)>0$. Let $\tau_{3}$ be 
the analogous strategy for player II:

$$
\tau_{3}(x, y)=\frac{\int_{A_{l}} \tau\left(x^{\prime}, y^{\prime}\right) d F_{2}\left(x^{\prime}, y^{\prime}\right)}{F_{2}\left(A_{l}\right)} \quad \text { if }(x, y) \in A_{l} .
$$

This is the probability that player II accepts $(x, y)$ if player I rejected it, and it is defined only for $(x, y) \in A_{l}$ such that $F_{2}\left(A_{l}\right)>0$. Suppose also that under $\sigma_{3}$ player I vetoes a candidate accepted by player II with the probability which is the conditional probability he would have vetoed it under $\sigma$ (conditional on $A_{l}$ ), and similarly for player II. By definition,

$$
\gamma_{2}(\sigma, \tau)=\gamma_{3}\left(\sigma_{3}, \tau_{3}\right) \quad \forall \sigma, \forall \tau
$$

Let now $\left(\sigma_{3}, \tau_{3}\right)$ be a subgame-perfect 0-equilibrium in $G_{3}$, which exists by assumption, since $\widehat{F}_{2}$ has a finite support. Let $\sigma^{*}$ and $\tau^{*}$ be the strategies that are defined as follows: as long as no player casts a veto, $\sigma^{*}$ follow $\sigma_{3}$ and $\tau^{*}$ follows $\tau_{3}$. Once player I (resp. player II) casts a veto, $\left(\sigma^{*}, \tau^{*}\right)$ coincides with a subgame-perfect $\frac{\varepsilon}{4}$-equilibrium in Markovian strategies in $G(i-1, j)$ (resp. in $G(i, j-1)$ ) with corresponding payoff $(u-, v+)$ (resp. $(u+, v-))$.

The strategies $\sigma^{*}$ and $\tau^{*}$ are Markovian. We now argue that $\left(\sigma^{*}, \tau^{*}\right)$ is a subgame-perfect $\varepsilon$-equilibrium in $G(i, j)$. We only prove that player I cannot gain more than $\varepsilon$ by deviating. Fixing $\tau^{*}$, player I's maximization problem reduces to a Markov decision problem, and therefore he has an optimal strategy $\sigma$ which is Markovian. Let $\sigma_{1}$ be the strategy that $\sigma$ induces on $G_{1}$, and let $\sigma_{3}$ be the corresponding discretized strategy defined above. Let $\sigma_{1}^{*}$ and $\tau_{1}^{*}$ be the strategies that $\sigma^{*}$ and $\tau^{*}$ induce in $G_{1}$, and let $\tau_{3}^{*}$ be the strategy defined in (5) w.r.t. $\tau^{*}$. Since $\sigma^{*}$ plays an $\frac{\varepsilon}{4}$-equilibrium once one of the players casts a veto, by $(3),(6)$, and since $\left(\sigma_{3}, \tau_{3}\right)$ is a subgame perfect 0-equilibrium in $G_{3}$ :

$$
\begin{aligned}
\gamma^{I}\left(\sigma, \tau^{*}\right) & \leq \gamma_{1}^{I}\left(\sigma_{1}, \tau_{1}^{*}\right)+\frac{\varepsilon}{4} \leq \gamma_{2}^{I}\left(\sigma_{1}, \tau_{1}^{*}\right)+\frac{\varepsilon}{2} \\
& =\gamma_{3}^{I}\left(\sigma_{1}, \tau_{1}^{*}\right)+\frac{\varepsilon}{2} \leq \gamma_{3}^{I}\left(\sigma_{1}^{*}, \tau_{1}^{*}\right)+\frac{\varepsilon}{2} \\
& =\gamma_{2}^{I}\left(\sigma_{1}^{*}, \tau_{1}^{*}\right)+\frac{\varepsilon}{2} \leq \gamma_{1}^{I}\left(\sigma_{1}^{*}, \tau_{1}^{*}\right)+\frac{3 \varepsilon}{4} \\
& \leq \gamma^{I}\left(\sigma^{*}, \tau^{*}\right)+\varepsilon
\end{aligned}
$$

so that indeed player I cannot gain more than $\varepsilon$ by deviating. 
The rest of this section is devoted to the proof that when $F_{2}$ has finite support a subgame-perfect 0-equilibrium exists. We recall that Flesch, Thuijsman and Vrieze (1997) proved that every two-player recursive absorbing game admits a stationary $\varepsilon$-equilibrium, yet, since our game has sequential moves, this result does not imply the existence of a SPE. The proof uses ideas from Vrieze and Thuijsman (1989) and Solan and Vieille (2002).

The proof is by induction on $i+j$. Assume that both $G(i, j-1)$ and $G(i-1, j)$ admit subgame-perfect 0-equilibrium in Markovian strategies, with corresponding payoffs $(u+, v-)$ and $(u-, v+)$ respectively. As before, $u-=$ -1 if $i=0$, and $v-=-1$ if $j=0$.

Let $a(x, y)$ and $b(x, y)$ be defined as in (1) and (2). Recall that the support $W$ of $F_{2}$ is finite. Define an auxiliary recursive absorbing game as follows.

- The set of actions (one-stage strategies) of player I is the finite set $S$ of all functions $s: W \rightarrow\{a, r\}$. That is, before player I observes the realized type of that stage, he has to determine for each possible type in $W$ whether he will accept it or reject it.

- The set of actions (one-stage strategies) of player II is the finite set $T$ of all functions $t: W \rightarrow\{a, r\}$. That is, before player II observes the realized type of that stage (and before he observes the decision of player I for that type), he has to determine for each possible type in $W$ whether he will accept it or reject it, assuming player I reject it.

- The payoff: denote the realized type by $(x, y)$. If player I accepts the type, the game is absorbed and the payoff is $a(x, y)$. If player I rejects the type and player II accepts it, the game is absorbed and the payoff is $b(x, y)$. If both players reject the type, the game continues to the next stage.

A mixed action for player I is a probability distribution over pure actions. By Kuhn's theorem it is equivalent to a function $\sigma: W \rightarrow[0,1]$ that assigns to each type $(x, y)$ the probability that it will be accepted, which is denoted by $\sigma(x, y) \in[0,1]$. Mixed actions $\tau$ for player II are defined analogously. Every mixed action is identified with the stationary strategy that plays it at every stage. 
For every pair $(\sigma, \tau)$ of stationary strategies, the probability of per-stage absorption is

$$
p(\sigma, \tau):=\sum_{(x, y) \in W} F_{2}(x, y)(\sigma(x, y)+(1-\sigma(x, y)) \tau(x, y)) .
$$

The pair $(\sigma, \tau)$ is called absorbing if $p(\sigma, \tau)>0$, and non-absorbing otherwise. Denote by $\gamma(\sigma, \tau)$ the expected payoff under the pair of strategies $(\sigma, \tau)$. Then

$\gamma(\sigma, \tau)= \begin{cases}(0,0) & (\sigma, \tau) \text { is non-absorbing } \\ \frac{\sum_{(x, y) \in W} F_{2}(x, y)(\sigma(x, y) a(x, y)+(1-\sigma(x, y)) \tau(x, y) b(x, y))}{\sum_{(x, y) \in W} F_{2}(x, y)(\sigma(x, y)+(1-\sigma(x, y)) \tau(x, y))} & \text { otherwise. }\end{cases}$

The following lemma is known in the theory of stochastic games (see, e.g., Vrieze and Thuijsman (1989) for a variant):

Lemma 4 Let $\left(\sigma_{n}, \tau_{n}\right)_{n \in \mathbf{N}}$ be a sequence of strategy pairs that converges point-wise to a limit $(\sigma, \tau)$. If $(\sigma, \tau)$ is absorbing then

$$
\lim _{n \rightarrow \infty} \gamma\left(\sigma_{n}, \tau_{n}\right)=\gamma(\sigma, \tau)
$$

Let $\mathcal{S}$ and $\mathcal{T}$ be the sets of all mixed actions for the two players respectively. Let $\mathcal{S}_{\varepsilon}$ be the set of all mixed actions $\sigma$ of player II that satisfy the following condition:

- $\sigma(x, y) \in[\varepsilon, 1-\varepsilon]$ for each $(x, y) \in W$ : player I must accept every type with probability at least $\varepsilon$, and must reject every type with probability at least $\varepsilon$.

The condition that $\sigma(x, y) \geq \varepsilon$ ensures that whatever player II plays, the game is bound to be absorbed, and therefore the payoff is continuous over $\mathcal{S}_{\varepsilon} \times \mathcal{T}$; the condition that $\sigma(x, y) \leq 1-\varepsilon$ ensures that each candidate is rejected with positive probability, and so every decision point of player II is reached with positive probability: a best response of player II to a strategy in $\mathcal{S}_{\varepsilon}$ cannot use punishments that hurt player II.

Observe that $\mathcal{S}_{\varepsilon}$ is convex, compact, and non-empty (assuming $\varepsilon$ is small enough). Let $G_{\varepsilon}$ be the recursive absorbing game in which the mixed-action space of player I is $\mathcal{S}_{\varepsilon}$, and the mixed-action space of player II is $\mathcal{T}$ (only player I is restricted). 
For every $(\sigma, \tau) \in \mathcal{S}_{\varepsilon} \times \mathcal{T}$, the game is bound to be absorbed. Since $\mathcal{S}_{\varepsilon}$ and $\mathcal{T}$ are convex and compact, the standard argument of Shapley (1953) implies that the game $G_{\varepsilon}$ has a 0-equilibrium in stationary strategies.

Lemma 5 Let $\left(\sigma_{\varepsilon}, \tau_{\varepsilon}\right)$ be a stationary 0-equilibrium in $G_{\varepsilon}$. For every type $(x, y)$,

1. $\tau_{\varepsilon}(x, y)$ is a best reply given the two possible continuation payoffs $\left(b(x, y)\right.$ and $\left.\gamma\left(\sigma_{\varepsilon}, \tau_{\varepsilon}\right)\right)$ : it chooses an action that attains the maximum $\max \left\{b^{I I}(x, y), \gamma^{I I}\left(\sigma_{\varepsilon}, \tau_{\varepsilon}\right)\right\}$.

2. $\sigma_{\varepsilon}(x, y)$ is the best reply (in the range $[\varepsilon, 1-\varepsilon]$, for player I) between the two continuation payoffs $a(x, y)$ and $\tau_{\varepsilon}(x, y) b(x, y)+(1-$ $\left.\tau_{\varepsilon}(x, y)\right) \gamma\left(\sigma_{\varepsilon}, \tau_{\varepsilon}\right)$.

Proof. We only prove the first statement. An analogous argument proves the second statement. Since $\sigma_{\varepsilon}(x, y) \leq 1-\varepsilon$, the type $(x, y)$ has a positive probability to be realized. If $\tau_{\varepsilon}$ does not choose the higher continuation payoff, player II could gain by deviating.

Lemma 6 At least one of the following two statements hold:

1. There is a subgame-perfect 0-equilibrium in Markovian strategies in $G(i, j)$ that is supported by $(u+, v-)$ and $(u-, v+)$ if one of the players casts a veto;

2. There is a stationary 0-equilibrium $\left(\sigma_{\varepsilon}, \tau_{\varepsilon}\right)$ in $G_{\varepsilon}$ such that at least one player accepts at least one candidate with probability $1-\varepsilon$.

Proof. Let $\left(\sigma_{\varepsilon}, \tau_{\varepsilon}\right)$ be a stationary equilibrium in $G_{\varepsilon}$, and assume that statement $(2)$ does not hold: under $\left(\sigma_{\varepsilon}, \tau_{\varepsilon}\right)$ no player accepts any candidate with probability $1-\varepsilon$. Then in particular

$$
a^{I}(x, y) \leq \gamma^{I}\left(\sigma_{\varepsilon}, \tau_{\varepsilon}\right), \quad \forall(x, y) \in W .
$$

Indeed, if $a^{I}(x, y)>\gamma^{I}\left(\sigma_{\varepsilon}, \tau_{\varepsilon}\right)$, player I would have gained by accepting $(x, y)$ with the maximal probability he can, $1-\varepsilon$. Similarly,

$$
b^{I I}(x, y) \leq \gamma^{I I}\left(\sigma_{\varepsilon}, \tau_{\varepsilon}\right), \quad \forall(x, y) \in W .
$$


If there was a candidate $\left(x^{*}, y^{*}\right) \in W$ such that $a^{k}\left(x^{*}, y^{*}\right) \geq \gamma^{k}\left(\sigma_{\varepsilon}, \tau_{\varepsilon}\right)$ for $k=1,2$, then the strategy pair in which both players accept a candidate if and only if it is $\left(x^{*}, y^{*}\right)$ is a 0 -equilibrium in $G(i, j)$ in stationary strategies, and statement (1) holds. Indeed, by $(12) a^{I}(x, y) \leq \gamma^{I}\left(\sigma_{\varepsilon}, \tau_{\varepsilon}\right) \leq a^{I}\left(x^{*}, y^{*}\right)$ for every $(x, y) \in W$, so that player I cannot gain by deviating, and by (13) $b^{I I}(x, y) \leq \gamma^{I I}\left(\sigma_{\varepsilon}, \tau_{\varepsilon}\right) \leq b^{I I}\left(x^{*}, y^{*}\right)$ for every $(x, y) \in W$, so that player II cannot gain by deviating.

Thus, if statement (1) does not hold, then for every $(x, y) \in W$ one has:

$$
\begin{aligned}
a^{I}(x, y) & =\gamma^{I}\left(\sigma_{\varepsilon}, \tau_{\varepsilon}\right) \quad \Longrightarrow \quad a^{I I}(x, y)<\gamma^{I I}\left(\sigma_{\varepsilon}, \tau_{\varepsilon}\right), \\
b^{I I}(x, y) & =\gamma^{I I}\left(\sigma_{\varepsilon}, \tau_{\varepsilon}\right) \quad \Longrightarrow \quad b^{I}(x, y)<\gamma^{I}\left(\sigma_{\varepsilon}, \tau_{\varepsilon}\right) .
\end{aligned}
$$

These two equations, together with (12) and (13), imply that any convex combination of the vectors $\{a(x, y), b(x, y)\}_{(x, y) \in W}$ gives at least one player $k$ strictly less than $\gamma^{k}\left(\sigma_{\varepsilon}, \tau_{\varepsilon}\right)$, which contradicts (11). Therefore if statement (2) does not hold then statement (1) holds.

Suppose that statement $(2)$ in Lemma 6 holds. For every $\varepsilon>0$ let $\left(\sigma_{\varepsilon}, \tau_{\varepsilon}\right)$ be a stationary 0-equilibrium in $G_{\varepsilon}$ in which at least one player accepts at least one candidate with probability $1-\varepsilon$. By compactness, there is a sequence $\left(\varepsilon_{k}\right)_{k \in \mathbf{N}}$ that converges to 0 such that the sequence $\left(\sigma_{\varepsilon_{k}}, \tau_{\varepsilon_{k}}\right)_{k \in \mathbf{N}}$ converges to a limit $\left(\sigma_{*}, \tau_{*}\right)$. Since in either $\sigma_{\varepsilon_{k}}$ or in $\tau_{\varepsilon_{k}}$ one of the players accepts at least one candidate with probability $1-\varepsilon$, this carries to the limit: in $\left(\sigma_{*}, \tau_{*}\right)$ at least one of the players accepts at least one candidate with probability 1 . In particular, the strategy pair $\left(\sigma_{*}, \tau_{*}\right)$ is absorbing.

Lemma 7 The strategy pair $\left(\sigma_{*}, \tau_{*}\right)$, supported by subgame-perfect equilibria in Markovian strategies in the continuation games $G(i, j-1)$ and $G(i-1, j)$ with corresponding payoffs $(u+, v-)$ and $(u-, v+)$, is a subgame-perfect 0 equilibrium in stationary strategies in $G(i, j)$.

Proof. Denote $\gamma_{*}:=\lim _{k \rightarrow \infty} \gamma\left(\sigma_{\varepsilon_{k}}, \tau_{\varepsilon_{k}}\right)$ (if the limit does not exist, consider a subsequence of $\left(\varepsilon_{k}\right)_{k \in \mathbf{N}}$ for which this limit does exist). Since $\left(\sigma_{*}, \tau_{*}\right)$ is absorbing, by Lemma 4:

$$
\gamma_{*}=\lim _{k \rightarrow \infty} \gamma\left(\sigma_{\varepsilon_{k}}, \tau_{\varepsilon_{k}}\right)=\gamma\left(\sigma_{*}, \tau_{*}\right)
$$

We first show that $\left(\sigma_{*}, \tau_{*}\right)$ is a 0 -equilibrium in $G(i, j)$. Define for every strategy $\sigma$ and $k \in \mathbf{N}$ a strategy $\widehat{\sigma}_{k}$ as follows: 


$$
\widehat{\sigma}_{k}(x, y)= \begin{cases}\varepsilon_{k} & \sigma(x, y)<\varepsilon_{k}, \\ \sigma(x, y) & \sigma(x, y) \in\left[\varepsilon_{k}, 1-\varepsilon_{k}\right], \\ 1-\varepsilon_{k} & \sigma(x, y) \geq 1-\varepsilon_{k} .\end{cases}
$$

Note that $\widehat{\sigma}_{k} \in \mathcal{S}_{\varepsilon_{k}}$ and $\sigma_{*}=\lim _{k \rightarrow \infty} \widehat{\sigma}_{k}$.

Let $\sigma$ be an arbitrary strategy of player I. If $\left(\sigma, \tau_{*}\right)$ is absorbing than by Lemma 4 and since $\left(\sigma_{\varepsilon_{k}}, \tau_{\varepsilon_{k}}\right)$ is an equilibrium in $G_{\varepsilon_{k}}$,

$$
\gamma^{I}\left(\sigma, \tau_{*}\right)=\lim _{\varepsilon \rightarrow 0} \gamma^{I}\left(\widehat{\sigma}_{k}, \tau_{*}\right) \leq \lim _{\varepsilon \rightarrow 0} \gamma^{I}\left(\sigma_{\varepsilon_{k}}, \tau_{*}\right)=\gamma_{*}^{I}=\gamma^{I}\left(\sigma_{*}, \tau_{*}\right)
$$

If $\left(\sigma, \tau_{*}\right)$ is non-absorbing then

$$
\gamma^{I}\left(\sigma, \tau_{*}\right)=0 \leq \gamma^{I}\left(\sigma_{*}, \tau_{*}\right)
$$

In both cases player I cannot profit by deviating from $\sigma_{*}$.

Similarly,

$$
\gamma^{I I}\left(\sigma_{*}, \tau\right) \leq \gamma^{I I}\left(\sigma_{*}, \tau_{*}\right), \quad \forall \tau .
$$

To show that $\left(\sigma_{*}, \tau_{*}\right)$ is a subgame-perfect equilibrium it is left to show that no player can gain by deviating after a particular candidate $(x, y)$ is realized.

Suppose then that the candidate $(x, y)$ is chosen. $\sigma_{\varepsilon_{k}}(x, y)$ is the best reply of player I (in the range $[\varepsilon, 1-\varepsilon]$ ) given the two possible continuation payoffs $a(x, y)$ and $\tau_{\varepsilon_{k}}(x, y) b(x, y)+\left(1-\tau_{\varepsilon_{k}}(x, y)\right) \gamma\left(\sigma_{\varepsilon_{k}}, \tau_{\varepsilon_{k}}\right)$. By continuity, and since $\varepsilon \rightarrow 0$ and $1-\varepsilon \rightarrow 1, \sigma_{*}(x, y)$ is the best reply of player I (in the range $[0,1])$ given the two possible continuation payoffs $a(x, y)$ and $\tau_{*}(x, y) b(x, y)+\left(1-\tau_{*}(x, y)\right) \gamma\left(\sigma_{*}, \tau_{*}\right)$. By the definition of $\tau_{*}$, player II indeed chooses, under $\tau_{*}$, the best outcome for player II among $b(x, y)$ or $\gamma\left(\sigma_{*}, \tau_{*}\right)$. By $(17)$

$$
\begin{aligned}
\gamma^{I}\left(\sigma, \tau_{*} \mid x, y\right) & =\max \left\{a^{I}(x, y), \tau_{*}(x, y) b^{I}(x, y)+\left(1-\tau_{*}(x, y)\right) \gamma^{I}\left(\sigma, \tau_{*}\right)\right\} \\
& \leq \max \left\{a^{I}(x, y), \tau_{*}(x, y) b^{I}(x, y)+\left(1-\tau_{*}(x, y)\right) \gamma^{I}\left(\sigma_{*}, \tau_{*}\right)\right\} \\
& =\gamma^{I}\left(\sigma_{*}, \tau_{*} \mid x, y\right)
\end{aligned}
$$

so that player I cannot profit by deviating after $(x, y)$ is realized.

We verify that an analog inequality holds for player II. $\tau_{\varepsilon_{k}}(x, y)$ is a best reply given the two possible continuation payoffs $\left(b(x, y)\right.$ and $\left.\gamma\left(\sigma_{\varepsilon_{k}}, \tau_{\varepsilon_{k}}\right)\right)$ : it chooses the action that attains the maximum $\max \left\{b^{I I}(x, y), \gamma^{I I}\left(\sigma_{\varepsilon_{k}}, \tau_{\varepsilon_{k}}\right)\right\}$. By continuity, $\tau_{*}(x, y)$ is a best reply given the two continuation payoffs 
$\left(b(x, y)\right.$ and $\left.\gamma\left(\sigma_{*}, \tau_{*}\right)\right)$ : it chooses the action that attains the maximum $\max \left\{b^{I I}(x, y), \gamma^{I I}\left(\sigma_{*}, \tau_{*}\right)\right\}$. Finally, for every strategy $\tau$, by (18),

$$
\begin{aligned}
\gamma^{I I}\left(\sigma_{*}, \tau \mid x, y\right) & =\max \left\{b^{I I}(x, y), \gamma^{I I}\left(\sigma_{*}, \tau\right)\right\} \\
& \leq \max \left\{b^{I I}(x, y), \gamma^{I I}\left(\sigma_{*}, \tau_{*}\right)\right\} \\
& =\gamma^{I I}\left(\sigma_{*}, \tau_{*} \mid x, y\right) .
\end{aligned}
$$

\section{Unexpected behavior of equilibria}

The number of vetoes each player has at the beginning of the game measures, in a sense, its relative strength. Indeed, Alpern and Gal (2008) proved that in a Markovian equilibrium, player I's (resp. player II's) expected payoff in $G(i, j)$ is always at least as much as his expected payoff after he casts a veto. In this section we provide several examples that challenge other common views of the role of vetoes in games.

If $F_{2}$ is symmetric, e.g., if $x$ and $y$ are independent with the same distribution $F$, then the game $G(0,0)$ always has a symmetric SPE in threshold strategies $\left(u_{0}, u_{0}\right)$, where $0<u_{0}<1$ is the threshold of acceptance: a candidate is accepted by a player if and only if her worth for that player is at least $u_{0}$ (see Alpern and Gal, 2008). Simple as it looks, the game $G(0,0)$ displays some unexpected behavior. We first show that the number of equilibria is not bounded.

Example 8 Let $n$ be a natural number. We construct a game $G(0,0)$ with $n$ threshold equilibria. Set

$$
z_{0}=0, z_{i}=1 /(2+\varepsilon)^{n-i}, i=1,2 \ldots, n .
$$

Let $x$ and $y$ be i.i.d. random variables. with a discrete distribution: their support is $\left\{z_{0}, z_{1}, \ldots, z_{n}\right\}$. The probabilities $p\left(z_{i}\right)=P\left(x=z_{i}\right)=P\left(y=z_{i}\right)$ satisfy

$$
p\left(z_{i}\right) \ll p\left(z_{i-1}\right), \quad i=1,2 \ldots, n .
$$

That is, the first positive score is almost certainly $z_{1}$, if not, then almost certainly $z_{2}$, etc. We show that all positive $z_{i}, i=1,2, \ldots, n$ can be threshold of symmetric equilibria. 
Let $1 \leq i \leq n$. If one player, say player $I$, uses a threshold strategy with threshold $z_{i}$ (so that he accepts a candidate only if his worth is at least $z_{i}$ ), then the payoff of each player is close to $z_{i} / 2$, and player II's best response is to use a threshold strategy with threshold $z_{i}$ as well. Indeed, if he uses a higher threshold then player I will almost surely stop before him, and his payoff will be close to 0 . If he uses a lower threshold than he would stop before player $I$, and his payoff would be close to $z_{i-1}$, which is smaller than $z_{i} / 2$.

It seems natural that the outcome is monotonic in the distribution of the population: if, say, one population is better than a second population, that is, if the distribution of the first population stochastically dominates the distribution of the second population, then the equilibrium payoff will be higher in the first population. The next example shows that this is not the case. This phenomenon is the well-known non-monotonicity of the equilibrium correspondence in one-shot games.

Example 9 Let $x, y$ be i.i.d. r.v.s. with the following three atom distribution:

$z=1$ with probability $\varepsilon$,

$z=d$ with probability $\varepsilon$, where $d \in(0,1)$ and

$z=0$ with probability $1-2 \varepsilon$.

We consider only SPE in threshold strategies, which always exists for $G(0,0)$ with a symmetric $F_{2}$, as mentioned above.

If $\frac{1}{3}<d<1$, the only such equilibrium is to accept a candidate whose worth for you is $d$ or 1 , and the corresponding equilibrium payoff to each player is (approximately) $(1+d) / 4$. If $0<d<\frac{1}{3}$ then, since $\left.d<(1+d) / 4\right)$, the only $S P E$ is to accept a candidate whose worth for you is 1 , and the equilibrium payoff to each player is (approximately) $1 / 2$. Since $\frac{1+d}{4}<\frac{1}{2}$ the SPE payoff for $0<d<\frac{1}{3}$ is higher than the unique SPE payoff for $\frac{1}{3}<d<1$ for both players.

\subsection{A Donation paradox}

The donation paradox was introduced by Fisher and Schotter (1978), who called it the "paradox of redistribution", in the context of power indices 
of simple voting games. This paradox occurs when a player gives an apparently valuable prerogative to another player, but 'does better,' according to some criterion. Specifically, when voting weights are reallocated, it may be observed that the voting power of some members, as measured by the Shapley-Shubik and Banzhaf power indices, increases while their voting weight decreases. (See also Felsenthal and Machover (1995), Kadane, Stone and Wallstrom (1999), and Holler and Napel (2004).) DeGroot and Kadane (1980) present an example of selecting a jury of two participants, in which one of the players prefers to give his veto right to the other player, because such a move yields a better outcome for himself. However, Theorem 3 in DeGroot and Kadane (1980) implies that if the (two) players have to choose only one candidate, a donation paradox cannot occur: giving away one of your vetoes to the other player lowers the payoff of a player. It should be noted, though, that the game presented by DeGroot and Kadane (1980) has a finite length, due to the rule of always accepting a candidate that was not vetoed. This property simplifies the situation in their model. On the other hand, in our model, it is possible that both players reject a candidate and the next candidate is called for. This introduces the inherent complexity of repeated games into our model.

As we show now, a donation paradox may occur in our model. Specifically, we present an example in which Player I prefers $G(0,1)$ to $G(1,0)$.

Example $10 F_{2}$ has 3 types of candidates, as follows:

\begin{tabular}{c|c} 
Type & Probability \\
\hline$(b, b)$ & $1-2 \varepsilon$ \\
$(1,0)$ & $\varepsilon$ \\
$(1-\varepsilon, 1-\varepsilon)$ & $\varepsilon$ \\
\hline
\end{tabular}

where $1 / 2<b<1$ and $\varepsilon \ll 1$.

Consider an equilibrium in $G(0,0)$. Player I accepts the type $(1,0)$, since this type has highest worth for him, ${ }^{1}$ and for an analog reason player II accepts the type $(1-\varepsilon, 1-\varepsilon)$. Since the average of these two vectors gives player II a payoff $\frac{1-\varepsilon}{2}<b$, player II accepts the type $(b, b)$. Therefore the

\footnotetext{
${ }^{1}$ We shorten "player I accepts candidates having type $(x, y)$ " to "player I accepts type $(x, y)$ ".
} 
unique equilibrium payoff, $\gamma_{0,0}$, is:

$$
\begin{aligned}
& \gamma_{0,0}^{I}=(1-2 \varepsilon) b+\varepsilon(1-\varepsilon)+\varepsilon=b+\varepsilon(2-2 b-\varepsilon)>b, \\
& \gamma_{0,0}^{I I}=(1-2 \varepsilon) b+\varepsilon(1-\varepsilon)=b+\varepsilon(1-2 b-\varepsilon)<b .
\end{aligned}
$$

Consider an equilibrium in $G(1,0)$. As in $G(0,0)$, player $I$ accepts the type $(1,0)$. Since $\gamma_{0,0}^{I}>b$ player I will veto $(b, b)$, and since $\gamma_{0,0}^{I I}>\frac{1-\varepsilon}{2}$ player II accepts the type $(b, b)$. It follows that if the type $(1-\varepsilon, 1-\varepsilon)$ is not accepted, the expected payoff for both players is close to $b$, and therefore both players will accepts the type $(1-\varepsilon, 1-\varepsilon)$. Thus, the unique equilibrium payoff, $\gamma_{1,0}$, is:

$\gamma_{1,0}^{I}=(1-2 \varepsilon) \gamma_{0,0}^{I}+\varepsilon(1-\varepsilon)+\varepsilon=b+\varepsilon(4-2 b+\varepsilon(-6+2 \varepsilon+4 b))>\gamma_{0,0}^{I}$,

$\gamma_{1,0}^{I I}=(1-2 \varepsilon) \gamma_{0,0}^{I I}+\varepsilon(1-\varepsilon)=b+\varepsilon(2-4 b+\varepsilon(-4+2 \varepsilon+4 b))<\gamma_{0,0}^{I I}$.

Consider now an equilibrium in $G(0,1)$. As in $G(0,0)$ player II will accept $(1-\varepsilon, 1-\varepsilon)$. Since $\gamma_{0,0}^{I I}<b$, player II will not veto $(b, b)$; Since $b$ is the minimal payoff to player $I$, it follows that player I will reject $(b, b)$. Since $\gamma_{0,0}^{I I}>0$ player II will veto $(1,0)$. Finally, player I will reject $(1,0)$, since by accepting it he will get $\gamma_{0,0}^{I}$, whereas by rejecting it he will get $1-\varepsilon$. Therefore the unique equilibrium is that both players accept only $(1-\varepsilon, 1-\varepsilon)$, and the corresponding equilibrium payoff is $\gamma_{0,1}=(1-\varepsilon, 1-\varepsilon)$.

As we see, player I's expected payoff in $G(0,1)$ is higher than his expected payoff in $G(1,0)$, so that a donation paradox occurs: player I prefers to give his veto to player II.

The donation paradox presented above cannot occur when $F_{2}$ is symmetric, and, in particular, when $x$ and $y$ are i.i.d.

Theorem 11 Assume that $F_{2}$ is symmetric. If the $S P E$ of $G(1,0)$ is unique, then player I never prefers $G(0,1)$ to $G(1,0)$.

Proof. Denote the unique equilibrium payoff of $G(1,0)$ by $\left(\gamma_{1,0}^{I}, \gamma_{1,0}^{I I}\right)$. By Alpern and Gal (2008, Theorem 9), there in a symmetric threshold equilibrium in $G(0,0)$. As we have seen in the proof of Theorem 2, every equilibrium payoff in $G(0,0)$ can be extended into a Markovian SPE in $G(1,0)$. Since there is a unique equilibrium payoff in $G(1,0)$, it follows that we can assume that in the equilibrium in $G(1,0)$ the continuation payoff is $G(0,0)$ is symmetric. 
One can verify that when one of the players does not have any veto rights, the order in which players play is irrelevant: by properly re-defining the strategies, an equilibrium in $G(0,1)$ can be turned into an equilibrium in $G(1,0)$. It follows that the unique equilibrium payoff in $G(0,1)$ is $\left(\gamma_{1,0}^{I I}, \gamma_{1,0}^{I}\right)$. We now show that $\gamma_{1,0}^{I} \geq \gamma_{1,0}^{I I}$.

Assume to the contrary that $\gamma_{1,0}^{I}<\gamma_{1,0}^{I I}$. We prove that if a candidate $(x, y)$ with $x<y$ is accepted with positive probability (and not vetoed by player I), then the candidate $(y, x)$ is accepted with probability 1 . Since $F_{2}$ is symmetric, and the continuation payoff if player 1 casts a veto is symmetric, this will imply that $\gamma_{1,0}^{I} \geq \gamma_{1,0}^{I I}$, a contradiction. Now, if such a candidate $(x, y)$ is accepted with positive probability by player I then

$$
y>x \geq \gamma_{1,0}^{I},
$$

so that player I accepts $(y, x)$ with probability 1 . If the candidate is accepted with positive probability by player II (and not vetoed by player I) then

$$
y>x \geq \gamma_{1,0}^{I I}>\gamma_{1,0}^{I},
$$

so that $(y, x)$ is accepted with probability 1 by player $\mathrm{I}$.

\section{Behavior in equilibrium}

In general, for every continuation payoffs $(u+, v-)$ and $(u-, v+)$ there may be many equilibria in $G(i, j)$. In this section we single out two types of equilibria, progressive equilibria and helpful equilibria. These equilibria represent the typical behavior of the players (see Remark 14 in this section). They also help us explain why one of the players may prefer that the other player would become more powerful in some situations, such as the donation paradox which occurs in the example provided in Section 4.1.

Suppose that $G(i-1, j)$ has an equilibrium with values $(u-, v+)$ and that $G(i, j-1)$ has an equilibrium with values $(u+, v-)$.

Definition 12 A Markovian subgame perfect equilibrium in $G(i, j)$ is called progressive if $u \leq u+, v \leq v+$, and (in case of equality) players choose to play the game in which the other player loses a veto rather than repeat the game $G(i, j)$. 
In a progressive equilibrium a player does not profit from the fact that the other player has one extra veto, and therefore he tries to make the other player use one veto.

Given the values $u-, u, v-, v$ it is easy to derive the SPE strategies as a function of the candidate values $x$ and $y$, depending on which of the five partitioning rectangles $\mathrm{A}, \mathrm{B}, \mathrm{C}, \mathrm{D}, \mathrm{E}$ in Figure 2 the point $(x, y)$ belongs to. (To simplify the discussion of ties, we assume these rectangles contain their left and bottom sides.)

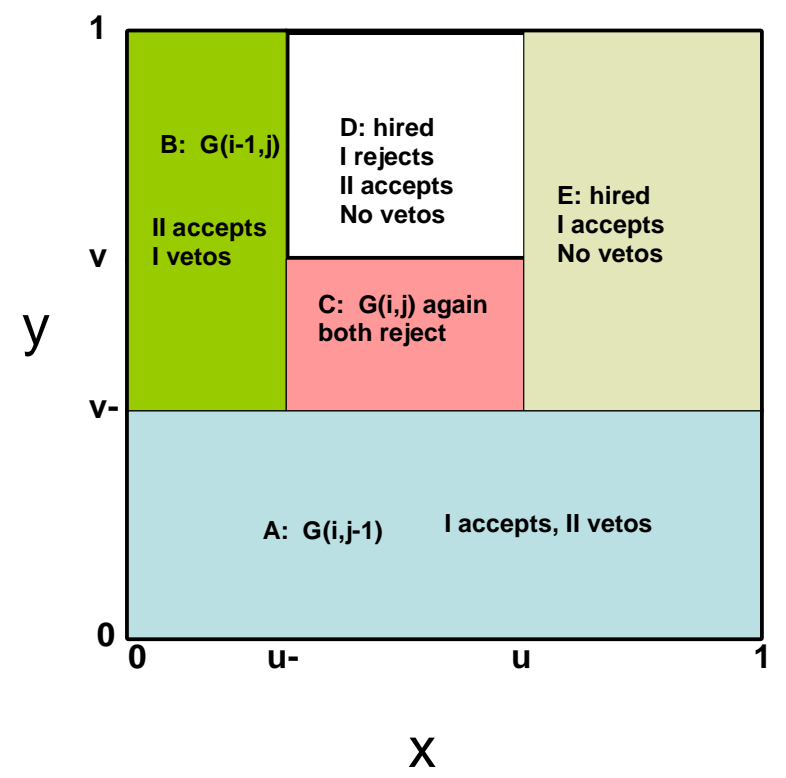

Figure 2. Progressive equilibrium for $G(i, j)$

Consider Figure 2, and suppose $y<v-$, that is, $(x, y) \in A$. Such a candidate will never be hired, because player II will cast a veto if necessary. The best player I can do is force player II to cast a veto. He does this by accepting the candidate. Similarly, if $(x, y) \in B$, then player I will cast a veto if necessary. The best player II can do is to accept the candidate, thereby forcing player I to cast a veto. If $(x, y) \in C$, neither player would cast a veto, and both prefer rejecting the candidate and replaying the game. If $(x, y) \in D \cup E$, the candidate will be hired, as at least one player prefers this to replaying $G(i, j)$, and neither player is willing to cast a veto. Denote by $(u, v)$ the 
equilibrium payoff in $G(i, j)$. Then

$$
\begin{aligned}
(u, v)= & T(u, v), \text { where } \\
T(u, v) \equiv & F_{2}(A) \cdot(u+, v-)+F_{2}(B) \cdot(u-, v+)+F_{2}(C) \cdot(u, v) \\
& +\int_{D \cup E}(x(z), y(z)) d F_{2}(z)
\end{aligned}
$$

Here, for every subset $H \subseteq[0,1]^{2}, F_{2}(H)$ is the probability that $(x, y) \in H$. If $F_{2}$ is the uniform distribution, then Lemma 16 of Alpern and Gal (2008) shows that, for any game $G(i, j), T$ is a contraction map of the unit square, which implies the existence of a unique progressive equilibrium.

Note that if $u<u+$ and $v<v+$, then the equilibrium actions played in the interior of each of the sets $A, B, C, D$ and $E$ in Figure 2 are strictly dominant.

In order to illustrate the other types of equilibrium we first analyze the game $G(1,0)$ for a symmetric $F_{2}$ (this includes the case in which $x$ and $y$ are i.i.d.). The game $G(0,0)$ has been analyzed in Alpern and Gal (2008). They proved that if $F_{2}$ is symmetric, then there exists a symmetric equilibrium. Denote the expected payoff to each player in $G(0,0)$ by $u_{0}$. We now consider the possibility of an equilibrium for $G(1,0)$ with equilibrium values $(u, v)$ with $u \geq u_{0}$ and $v \geq u_{0}$. Previously we had assumed an extra veto for player I would hurt player II, with $v<u_{0}$. If $v>u_{0}$, player II would rather replay $G(1,0)$ and get $v$ than go to $G(0,0)$ and get $u_{0}$. So player I will never use his veto, as in such a case player II would previously have rejected the candidate. We call such an equilibrium helpful. (To simplify the discussion of ties, we assume these rectangles contain their left and bottom sides.) 


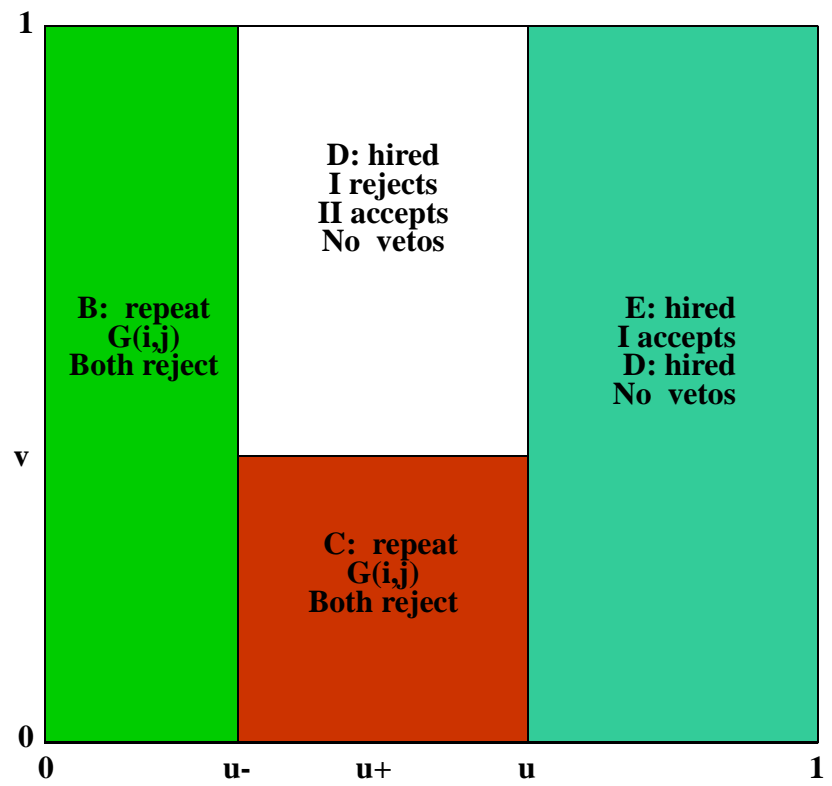

Figure 3. A helpful equilibrium for $G(1,0)$

We now consider the general situation.

Definition 13 An equilibrium in $G(i, j)$ is called helpful if $u>u+$ and $v>v+$.

An equilibrium is helpful if each player loses when the other player casts a veto. In such a case, in equilibrium a player will not accept a candidate that the other player does not like, so that the other player will not be forced to use his veto power.

A helpful equilibrium has the profile depicted in Figure 4. (To simplify the discussion of ties, we assume these rectangles contain their left and bottom sides. Also, player II accepts a candidate in $\mathrm{E}$ if $y \geq v$ and rejects her if $y<v)$ : 


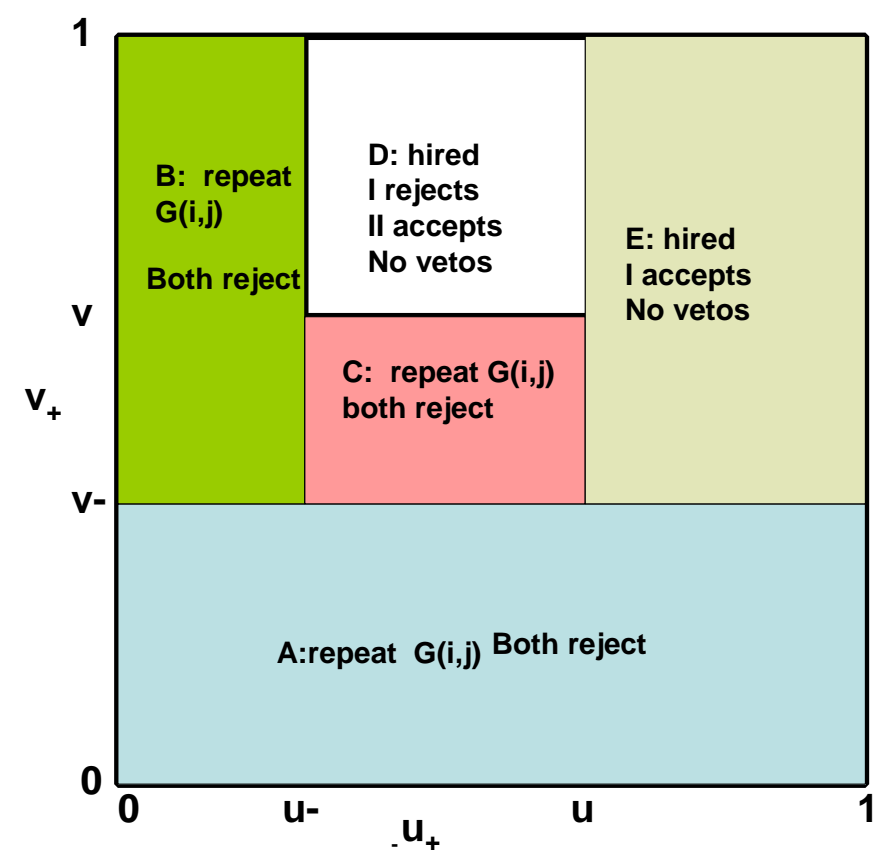

Figure 4. A helpful equilibrium for $G(i, j)$

Indeed, consider a candidate $(x, y)$. If $y<v$ - player II will cast a veto. Since $u<u+$ player I prefers that player II would not cast a veto, hence he will reject such a candidate (region A in Figure 4). For an analog reason both players reject the candidate if $(x, y) \in B$. The behavior of the players in regions $\mathrm{C}, \mathrm{D}$ and $\mathrm{E}$ is as in a progressive equilibrium. Thus, the payoff $(u, v)$ that corresponds to an helpful equilibrium is a fixed point

$$
(u, v)=T(u, v),
$$

where

$$
T(u, v)=F_{2}(A \cup B \cup C) \cdot(u, v)+F_{2}(D \cup E) \cdot\left(E_{1}(D \cup E), E_{2}(D \cup E)\right) .
$$

Here $E_{i}(D \cup E), i=1,2$, are the conditional expectations of $x$ and $y$ in $(D \cup E)$.

Note that if $u>u+$ and $v>v+$, then the actions in each region in Figure 4 are strictly dominant. 
There can also be a mixed equilibrium, in which $u<u_{+}$but $v>v_{+}$or $u>u_{+}$but $v<v_{+}$.

Back to the donation paradox presented in Section 4.1, we note that in $G(1,0)$ the unique Markovian SPE is progressive $(v<v+$ so that player II will choose $(b, b)$ and force player I to use his veto). On the other hand, in $G(0,1)$ the unique Markovian SPE is helpful $(u>u+$ so that player I will not accept the candidate $(1,0)$ that will make player II use his veto). This explains why player I prefers $G(0,1)$ over $G(1,0)$.

Remark 14 Note that if a 'degeneracy', $u=u+$ or $v=v+$, does not occur, then, for any game $G(i, j), i, j \geq 0$, with an absolutely continuous $F_{2}$, there exist only pure equilibria (possibly more than one) which is either progressive or helpful or mixed. For example, assume that we found an equilibrium payoff $(u, v)$ (either by a numerical scheme based on Section 3, or by some theoretical analysis), and it turns out that $u<u+$ and $v<v+$. Then the only possible equilibrium strategies, are given by the progressive equilibrium in Figure 2, because the actions in this figure are strictly dominant. Similarly, if $u>u+$ and $v>v+$, then the only possible equilibria are given by helpful equilibria as in Figure 4, because the actions in this figure are strictly dominant. The mixed cases can be handled in a similar way.

\subsection{A simple three atom i.i.d. distribution}

In this section we illustrate the structure of the progressive and the helpful equilibria in $G(1,0)$ for a simple three atom i.i.d. distribution. The existence of a helpful equilibrium in $G(1,0)$ is surprising because we proved in Theorem 11 that if $x$ and $y$ are $i . i . d$. and the equilibrium in $G(1,0)$ is unique then player I never prefers $G(0,1)$ to $G(1,0)$.

Notation $15 A$ simple three atom distribution is a distribution in which $x$ and $y$ are i.i.d. with three atoms of equal probability, $1 / 3$, at 0,1 and $b$, where $0<b<1$.

This distribution is quite natural with the score being either 'good' or 'bad' or 'medium'. Yet, it may lead to unexpected results for $G(1,0)$.

It should be noted that since $F_{2}$ has an atom at $(1,1)$, then there exists a SPE for $G(0,0)$ (and for $G(i, j)$ in general) in which only the candidate 
$(1,1)$ is accepted. This SPE will be denoted as $\{(1,1)\}$. This SPE is not 'stable' in the sense that introducing a discount factor of $1-\varepsilon$ makes it disappear' ${ }^{2}$.

If $F_{2}$ is symmetric, then in $\boldsymbol{a n y}$ other SPE each player always accepts a candidate whose worth (to him) is 1 (because in such SPE his payoff is strictly smaller than 1).

Theorem 16 Under the simple three atom distribution with $3 / 4<b<1$ for the game $G(0,0)$. Except of the SPE $\{(1,1)\}$, the only SPE is the one in which each player accepts a candidate if and only if its worth for him is $b$ or 1.

Proof. As we have noted, in any SPE, which is different from $\{(1,1)\}$, each player always accepts a candidate if its worth to him is 1 , so the five candidates $(1,1),(1, b),(1,0),(b, 1)$, and $(0,1)$ are always accepted. Consider any (mixed) SPE and let the probability of acceptance of $(b, b),(b, 0)$ and $(0, b)$ be denoted by $p_{b b}, p_{b 0}$ and $p_{0 b}$. Then, the expected payoff to player I satisfies

$$
u=\frac{3+b+b p_{b b}+b p_{b 0}}{5+p_{b b}+p_{b 0}+p_{0 b}}<b
$$

(because $b>3 / 4$ ). Thus, the only possibility for a SPE is for player I to always accept candidates $(b, b)$ and $(b, 0)$.

Similarly, the only possibility for a SPE is for player II to always accept candidates $(b, b)$ and $(0, b)$.

If both players use the threshold $b$, then all the candidate types except $(0,0)$ are hired. Thus, the expected payoff for both players under this policy is the average score of the other eight types, so that, since $b>\frac{3}{4}$

$$
u_{0}=\frac{3+3 b}{8}<b \text {. }
$$

We now analyze the game $G(1,0)$ assuming that the above SPE is used for $G(0,0)$. We present the following SPE for $G(1,0)$ which yields expected payoff strictly more than $u_{0}$ for both players. This is obtained by the following

\footnotetext{
${ }^{2}$ Because, e.g., player I would also accept $(1,0)$ and $(1, b)$ which give him an immediate payoff equal to 1 .
} 
'helpful' strategies. Player I accepts 1 , rejects, but not vetoes, $b$, and vetoes 0 . Player II accepts if and only if both $x$ and $y$ are at least $b$. Thus, player II acts in such a way that player I never vetoes.

Under the helpful strategies, the following (equally likely) candidates $(b, b),(b, 1),(1,0),(1, b)$, and $(1,1)$ are hired. All the other candidates are rejected and the game repeats itself. Thus, the expected payoff to player I is

$$
u=\frac{3+2 b}{5}>b
$$

and since $b>\frac{3}{4}$, the expected payoff to player II is

$$
v=\frac{2+2 b}{5}<b
$$

Inequality (27) implies that player I cannot improve by accepting candidates with $y=b$. On the other hand, by (28) and (26), $v>u_{0}$, so player II cannot improve by accepting candidates with $x=0$, forcing player I to veto. Thus,

Theorem 17 If $b>3 / 4$, then the helpful strategies form a SPE for $G(1,0)$, which yields payoffs strictly larger than $u_{0}$ for both players.

This equilibrium will be denoted by 'helpful SPE'.

Example 18 If $b=.9$, then $u_{0}=.71$. In the game $G(1,0)$ the helpful strategies lead to payoffs $u=.96$, and $v=.76$.

In addition to this SPE there exists a progressive SPE for $G(1,0)$ in which player $I I$ accepts $x=0$ (and $y \geq b$ ) and player $I$ accepts $x \geq b=.9$, with payoffs $u=.87$ and $v=.65$.

We now show that in some cases only helpful strategies exist.

Theorem 19 Progressive equilibrium for $G(1,0)$ need not exist.

Proof. Take the three atoms model in which $b$ satisfies

$$
\frac{3}{4}<b<\frac{11}{13}
$$

We now show that (except of the SPE $\{(1,1)\}$ ) the helpful SPE described above is the only equilibrium for $G(1,0)$. 
In any equilibrium (different from $\{(1,1)\}$ ) player I always accepts $x=1$ and vetoes $x=0$. It follows from (26) that if $x=b$, then 'veto' cannot be an optimal action for player I. We now show, by contradiction, that under (29) the only possibility for an equilibrium is that player I rejects $x=b$. Suppose that player I accepts $x=b$, then player II's best response is to accept $x=0$ forcing a veto by player I (because rejecting $x=0$ leads to an expected payoff $(2+2 b) / 6<u_{0}$ by $\left.(26)\right)$. So, the expected payoff to player I would be $\left(u_{0}+b+1\right) / 3$ which is greater than $b$ by (26) and (29). Thus, player I can improve by rejecting $x=b$, which leads to a contradiction. Thus, in the interval (29) player I's best decision for $x=b$ is to reject it, so the only equilibrium, which is also subgame perfect, is the the helpful equilibrium.

For example, if $b=.8$, then $u_{0}=.675$, and the only equilibrium in $G(1,0)$ leads to payoffs $u=.92$, and $v=.72$.

Lemma 20 For any $0<b<1$, the expected payoff, $u$, to player $I$ in any helpful SPE of $G(1,0)$ exceeds $b$.

Proof. If player II rejects candidates with $x=0$, then, only candidates with $x=b$, or 1 can be accepted so, obviously, $u>b$.

If $b$ is close to 1, then, as in Example 18 the progressive SPE leads to $u<b$.

Remark 21 In the game $G(2,0)$ it is also it is possible to have both progressive and helpful SPE. In the simple three atom distribution for any equilibrium, player $I$ accepts $x=1$ and vetoes $x=0$. On the other hand, contrary to $G(1,0)$, it seems that all the three actions are possible for $x=b$ in SPE. For example, playing $G(2,0)$ with $b=.9$, if player I uses the progressive $S P E$ for $G(1,0)$, then he would not veto $x=b$, but if he uses the helpful SPE, then it follows from Lemma 20 that he would veto her.

Remark 22 Assume that $x, y$ are i.i.d with any distribution. Then $u_{0}>$ $E(x)$ and for $G(\infty, 0)$ the payoff to player $I$ is 1 and to $I I E(x)$. It is easily seen that the payoff to player I in $G(n, 0)$ is monotonic increasing as a function of $n$. On the other hand, if helpful equilibrium exists for some $n$, then the payoff to player II is not monotonic in $n$. 


\section{Bibliography}

Alpern, S., and Gal., S. (2008). Analysis and design of selection committees: A game Theoretic secretary problem. LSE-CDAM-2008-03

Brams, S. J. and Davis, M. D. (1976). A game-theory approach to jury selection. Trial 12, 47-49.

Brams, S. J. and Davis, M. D. (1978). Optimal jury selection: A Game theoretic model for the exercise of peremptory challenges. Operations Research 26, 966-991.

Bruss, F. T., Drmota, M.. and Louchard, G. (1998) The complete solution of the competitive rank selection problem, Algorithmica 22, 413-447.

DeGroot, M. H., and Kadane, J. B. (1980). Optimal challenges for selection. Operations Research, 28, 952-968

Enns, E. G., and Ferenstein, E. Z. (1978) On a multi-person time-sequential game with priorities. In Sequential analysis, design methods and applications, Taylor and Francis.

Eriksson, K., Sjöstrand, J. and Strimling, P. (2007). Optimal expected rank in a two-sided secretary problem, Operations Research, in press.

Felsenthal, D., and Machover, M. (1995). Postulates and paradoxes of relative voting power - A critical re-appraisal, Theory and Decision, 38, 195-229.

Ferguson, T. (1989). Who solved the secretary problem? Statist. Sci. 4, $282-296$.

Ferguson, T. (2001). Selection by Committee. eScholarship Repository, University of California. http:repositories.edlib.org/uclastat/papers/2001010108.

Flesch, J., Thuijsman, F., and Vrieze, K. (1996) Recursive repeated games with absorbing states, Mathematics of Operations Research, 21, 1016-1022,

Fisher, D. and Schotter, A. (1978), The inevitability of the "paradox of redistribution" in the allocation of voting weights, Public Choice, 33, 49-67.

Holler, M.J., and Napel, S. (2004). Monotonicity of power and power measures. Theory and Decision, 56, 93-111.

Kadane, J. B., Stone, C. A. and Wallstrom, G. (1999). The donation paradox for peremptory challenges, Theory and decision, 47, 139-151.

Mertens, J.F. (1987) Repeated games, Proceedings of the International Congress of Mathematicians, Berkeley, California, 1528-1577.

Ramsey, D., and Szajowski, K. (2005). Bilateral approach to the secretary problem. Advances in dynamic games: Applications to economics, edited by A. S. Nowak and K. Szajowski, 271-284. 
Roth, A., Kadane, J. B., and DeGroot, M. H. (1977). Optimal peremptory challenges in trials by juries: A bilateral sequential process. Operations Research 25, 901-919.

Sakaguchi, M., (2005). Optimal stopping games where players have weighted privilege, Advances in dynamic games: Applications to economics, edited by A. S. Nowak and K. Szajowski, 285-294.

Shapley L.S. (1953). Stochastic Games, Proc. Nat. Acad. Sci. U.S.A. 1095-1100.

Solan, E., and Vieille, N. (2002), Correlated Equilibrium in Stochastic Games, Games and Economics Behavior, 38, 362-399.

Vrieze, O. J., and Thuijsman, F. (1989).On equilibria in repeated games with absorbing states. International Journal of Game Theory, 18, 293-310. 